Research, Society and Development, v. 9, n. 12, e36491211376, 2020

(CC BY 4.0) | ISSN 2525-3409 | DOI: http://dx.doi.org/10.33448/rsd-v9i12.11376

\title{
Limiares hedônicos para redução de cloreto de sódio em biscoito salgado ${ }^{1}$ \\ Hedonic thresholds for sodium chloride reduction in cracker \\ Umbrales hedónicos para la reducción del cloruro de sodio en galleta salada
}

Recebido: 19/12/2020 | Revisado: 19/12/2020 | Aceito: 21/12/2020 | Publicado: 27/12/2020

\section{Laudiane Justo Sant'anna}

ORCID: https://orcid.org/0000-0001-6296-0777

Universidade Federal do Espírito Santo, Brasil

E-mail:laudianejusto@gmail.com

Cirila Ionara Almeida Araújo

ORCID: https://orcid.org/0000-0002-1333-3512

Universidade Federal do Espírito Santo, Brasil

E-mail: ionaracirila@yahoo.com.br

Lara Lima Cornejo

ORCID: https://orcid.org/0000-0002-2650-9839

Universidade Federal do Espírito Santo, Brasil

E-mail: lara.cornejo@hotmail.com

Eduardo da Silva Moreira

ORCID: https://orcid.org/0000-0003-1548-8566

Universidade Federal do Espírito Santo, Brasil

E-mail: edumoasilva@gmail.com

Matheus Custódio de Paula

ORCID: https://orcid.org/0000-0001-7103-8186

Universidade Federal do Espírito Santo, Brasil

E-mail: matheuscustodiop95@gmail.com

Raquel Viera de Carvalho

ORCID: https://orcid.org/0000-0001-7329-9750

Universidade Federal do Espírito Santo, Brasil

E-mail: raquelvcarvalho@ hotmail.com

\footnotetext{
${ }^{1}$ Trabalho apresentado no CBCP 2020 - Congresso on-line Brasileiro de Tecnologia de Cereais e Panificação, selecionado para publicação na forma de artigo completo.
} 
Suzana Maria Della Lucia

ORCID: https://orcid.org/0000-0003-0322-7684

Universidade Federal do Espírito Santo, Brasil

E-mail: smdlucia@yahoo.com.br

Tarcísio Lima Filho

ORCID: https://orcid.org/0000-0002-2794-5544

Universidade Federal do Espírito Santo, Brasil

E-mail: tarcisiolimaf@yahoo.com.br

\title{
Resumo
}

Um dos fatores responsáveis pelo alto índice de hipertensão na população mundial é a ingestão excessiva de sódio. Atualmente, este consumo é duas vezes maior do que o recomendado e, portanto, tem-se buscado maneiras de diminuir o teor desse componente dos alimentos, principalmente, dos industrializados. Objetivou-se investigar o quanto de cloreto de sódio é possível reduzir em biscoito tipo salgado sem comprometer sua aceitação (limiar de aceitação comprometida - LAC) e sem resultar em rejeição sensorial (limiar de rejeição hedônica - LRH). Por meio da Metodologia dos Limiares Hedônicos (MLH) foram determinados o LAC e o LRH, utilizando-se uma amostra controle com $1,81 \%$ de cloreto de sódio e amostras estímulo com concentrações de 1,80\%, 1,35\%,0,90\%, 0,45\% e 0,0\% de cloreto de sódio. Calculou-se o LAC de $0,84 \%$ de cloreto de sódio, demostrando que, somente a partir da redução de 53,59\% no teor de cloreto de sódio ocorreu redução da aceitação sensorial do biscoito. Além disso, verificou-se que apenas o biscoito sem adição de cloreto de sódio é rejeitado sensorialmente pelos consumidores. Dessa forma, os resultados comprovam que é possível uma redução gradativa do teor de sódio de biscoitos salgados sem suceder em menor aceitação por parte dos consumidores.

Palavras-chave: Limiares sensoriais; Limiar de aceitação comprometida; Limiar de rejeição hedônica; Redução de sódio; Redução de sal.

\begin{abstract}
One of the main factors responsible for the worldwide high hypertension rate is the excessive sodium intake. Currently, the population salt consumption is twice the recommended limit and thus initiatives to reduce this component content in food, especially industrialized products, have been targeted. In this context, this study aimed to investigate what amount of sodium chloride can be reduced from salted crackers without compromising its acceptance
\end{abstract}


(compromised acceptance threshold - CAT) neither causing its sensory rejection (hedonic rejection threshold - HRT). The Hedonic Thresholds Methodology (HTM) was applied to determine the CAT and HRT, where the standard stimulus sample had $1.81 \%$ of sodium chloride and the stimulus samples had $1.80 \%, 1.35 \%, 0.90 \%, 0.45 \%$ and $0.0 \%$ of sodium chloride. The CAT of $0.84 \%$ sodium chloride was calculated, demonstrating that only after reducing $53.59 \%$ in the sodium chloride content the sensorial acceptance of cracked is compromised. In addition, it was found that only the cracker without the addition of sodium chloride is sensorially rejected by consumers. The results indicate that it is possible to gradually reduce the sodium content of crackers without occurring in less acceptance by consumers.

Keywords: Sensory thresholds; Compromised acceptance threshold; Hedonic rejection threshold; Sodium reduction; Salt reduction.

\section{Resumen}

Uno de los factores responsables por la alta tasa de hipertensión en la población mundial es la ingesta excesiva de sodio. Actualmente, este consumo es el doble de lo recomendado y, por tanto, se han buscado formas de disminuir el contenido de este componente de los alimentos, especialmente los alimentos industrializados. El objetivo de este estudio fue investigar cuánto cloruro de sodio se puede reducir en galleta salada sin comprometer su aceptación (umbral de aceptación comprometida - UAC) y sin resultar en rechazo sensorial (umbral de rechazo hedónico - URH). Usando la Metodología de Umbral Hedónico (MUH), se determinó el umbral de aceptación comprometida (UAC) y el umbral de rechazo hedónico (URH), utilizando una muestra estándar con $1,81 \%$ de cloruro de sodio y muestras de estímulo con concentraciones de $1,80 \%, 1,35 \%, 0,90 \%, 0,45 \%$ y $0,0 \%$ de cloruro de sodio. El UAC calculado fue $0,84 \%$ de cloruro de sodio, demostrando que solo después de reducir $53,59 \%$ en el contenido de cloruro de sodio hubo una reducción en la aceptación sensorial de la galleta. Además, se constató que los consumidores solo rechazan sensorialmente las galletas sin la adición de cloruro de sodio. De esta forma, los resultados revelan que es posible reducir gradualmente el contenido de sodio en galletas saladas sin apuntar una menor aceptación por parte de los consumidores.

Palabras clave: Umbrales sensoriales; Umbral de aceptación comprometida; Umbral de rechazo hedónico; Reducción de sodio; Reducción de sal. 


\section{Introdução}

O sódio é um elemento essencial para o funcionamento do corpo humano, sendo necessário, por exemplo, para a manutenção do volume plasmático, equilíbrio ácido-base, transmissão de impulsos nervosos e função celular (WHO, 2020). No entanto, o consumo excessivo de sódio contribui para o aumento da pressão arterial, o principal fator de risco metabólico, em termos de mortalidade, associado às chamadas doenças crônicas não transmissíveis (Alburto et al., 2013; Mozaffarian et al., 2014). Essas incluem doenças cardiovasculares, respiratórias, cânceres, osteoporose, diabetes e insuficiência renal, que resultam não apenas em óbitos, mas também na incapacidade prematura no Brasil (WHO, 2018; Brasil, 2011a).

A média mundial de consumo de sal de cozinha (cloreto de sódio), fonte primária de sódio na alimentação, é de 9 a 12 gramas diárias por indivíduo, o que equivale a cerca de duas vezes o nível máximo recomendado (WHO, 2014). Preocupados com esta situação e suas consequências, os governos e Estados Membros da Organização Mundial da Saúde propuseram reduzir a ingestão de sódio pela população mundial em $30 \%$ até 2025 , tendo como uma das estratégias a diminuição do teor de cloreto de sódio $(\mathrm{NaCl})$ em alimentos industrializados (WHO, 2020). Com esse objetivo, o Ministério da Saúde do Brasil, juntamente com diversas associações de indústrias de alimentos, acordaram o compromisso em realizar a redução gradativa de sódio em diferentes alimentos comercializados no Brasil (Brasil, 2011b).

Apesar dos acordos propostos, a diminuição do teor de sódio em alimentos ainda gera insegurança por parte das indústrias, uma vez que o sal é um dos principais ingredientes responsáveis pela aceitação sensorial de muitos produtos. Sua redução pode causar respostas negativas pelo consumidor final e, consequente, perda de mercado. Portanto, é necessário realizar a redução do teor de sódio sem afetar a qualidade sensorial do alimento.

A Metodologia dos Limiares Hedônicos (MLH) é uma metodologia de análise sensorial que permite determinar o limiar de aceitação comprometida (LAC) e o limiar de rejeição hedônica (LRH), que representam, respectivamente, a intensidade de um estímulo na qual a aceitação sensorial de um produto passa a ser comprometida (LAC) e a intensidade de um estímulo em que começa a ocorrer rejeição sensorial (LRH) do alimento (Lima Filho et al., 2015, 2017, 2018). Por meio da MLH é possível determinar a partir de qual concentração, a redução de cloreto de sódio, resulta em comprometimento da aceitação sensorial (LAC) e rejeição sensorial (LRH) de alimentos (Lima Filho et al., 2019). Diante do exposto, objetivou- 
se determinar o quanto é possível reduzir de cloreto de sódio em biscoito salgado sem provocar a redução da aceitação sensorial (LAC) e o início da rejeição sensorial (LRH) do produto. O biscoito salgado foi escolhido por possuir elevada concentração de sódio e ser um alimento muito consumido no mundo, devido à sua praticidade e sabor, resultando no aumento do consumo de sódio pela população (Manley, 2010; Brasil, 2011b, 2015; Nielsen, 2014; ABIMAPI, 2017).

\section{Materiais e Métodos}

O presente estudo foi aprovado pelo Comitê de Ética em Pesquisa com Seres Humanos da Universidade Federal do Espirito Santo (UFES), sob o número de parecer 3.100.527. Foi realizada uma pesquisa laboratorial e experimental, na UFES.

\subsection{Elaboração dos biscoitos}

A formulação e a elaboração dos biscoitos foram realizadas de acordo com Davidson (2018), com adaptações. Os biscoitos foram preparados utilizando-se $100 \mathrm{~g}$ de farinha de trigo (Boa Sorte $\left.{ }^{\circledR}\right), 2,85 \mathrm{~g}$ de açúcar cristal (sacarose, marca Paineiras $\left.{ }^{\circledR}\right), 2,00 \mathrm{~g}$ de açúcar invertido (Art Alimentos ${ }^{\circledR}$ ), 6,02 g de extrato de malte (Art Alimentos®), 11,66 g de óleo de soja (Soya $\left.{ }^{\circledR}\right)$, 0,20 g de lecitina de soja (Art Alimentos $\left.{ }^{\circledR}\right), 1,84 \mathrm{~g}$ de fermento químico sal amoníaco (Art Alimentos ${ }^{\circledR}$ ); 2,16 g de bicarbonato de sódio, fosfato monocálcio, carbonato de cálcio (fermento químico Pó Royal®), 0,01 g de enzima protease (Bio lab®), 30mL de água e sal de cozinha (cloreto de sódio, marca Globo®), em concentrações variadas entre as amostras. Os ingredientes utilizados foram adquiridos na comércio local da cidade de Alegre/ES e em lojas especializadas em produtos para panificação.

O teor de cloreto de sódio da amostra controle $(1,81 \% \mathrm{~m} / \mathrm{m})$ foi determinado por meio de testes preliminares, para atender os pré-requisitos da MLH, e baseado na média aritmética do teor de sódio presente na tabela nutricional de diferentes biscoitos salgados comerciais.

Por meio da tabela nutricional, verificou-se o teor de sódio de biscoitos salgados comerciais brasileiros. Além do sal de cozinha (cloreto de sódio), dependendo da formulação do biscoito, pode existir outros ingredientes contendo sódio, como o bicarbonato de sódio e a água utilizada na formulação. No entanto, a contribuição desses outros ingredientes no teor de sódio total do produto é bem inferior ao do cloreto de sódio, que é a principal fonte de sódio em biscoitos salgados. Sendo assim, foi possível determinar apenas o teor aproximado de 
(CC BY 4.0) | ISSN 2525-3409 | DOI: http://dx.doi.org/10.33448/rsd-v9i12.11376

cloreto de sódio dos produtos comerciais, que serviu de referência para elaboração das amostras que foram analisadas sensorialmente, pelos próprios pesquisadores, nos testes preliminares.

Por meio dos testes preliminares, buscou-se atender os pré-requisitos da MLH, em que a amostra controle deve ser a de maior aceitação sensorial e deve ser estudada uma faixa de intensidade do estímulo (no caso, concentração de cloreto de sódio) que inclua os limiares hedônicos (LAC e LRH) a serem determinados, ou seja, deve existir ao menos uma amostra estímulo (no caso, amostra com redução de cloreto de sódio) que tenha aceitação sensorial semelhante à amostra controle e ao menos uma amostra estímulo que tenha menor aceitação sensorial quando comparada à amostra controle, para seja possível determinar o LAC, ou que seja rejeitada sensorialmente, para que seja possível determinar o LRH (Lima Filho et al., 2015; Lima Filho \& Minim, 2018).

Sendo assim, determinou-se a amostra controle contendo 1,81\% de cloreto de sódio e, a partir dessa concentração, elaborou-se amostras estímulo com concentrações de cloreto de sódio reduzidas $(1,80 \%, 1,35 \%, 0,90 \%, 0,45 \%$ e $0,0 \% \mathrm{~m} / \mathrm{m})$, que foram comparadas com a amostra controle na análise sensorial.

Para elaboração dos biscoitos, inicialmente, foi realizada a mistura dos ingredientes secos por 2 minutos (farinha de trigo, açúcar cristal, lecitina de soja, extrato de malte e enzima), exceto fermentos e o sal. Em seguida foram adicionados os ingredientes líquidos (óleo de soja, açúcar invertido e parte da água) sendo misturados por mais 5 minutos em misturadora (marca Arke, modelo MMX5X1). Após a mistura, foram adicionados os fermentos e, em segundo momento, o cloreto de sódio dissolvido em água, permitindo assim uma maior homogeneização do sal. Após a adição do sal, a massa foi misturada por mais 3 minutos e levada para fermentação, por 2,5 horas em estufa a $35^{\circ} \mathrm{C}$, e, em seguida, foi laminada em laminador para massas (marca Arke, modelo MMX5X1).

Os biscoitos foram modelados manualmente utilizando moldes redondos, possuindo, aproximadamente, $5 \mathrm{~cm}$ de diâmetro e $0,3 \mathrm{~cm}$ de espessura. Foram assados em forno com convecção de ar (marca Prática Technipan, modelo MINICONV VP) a $180^{\circ} \mathrm{C}$ por, aproximadamente, 6 minutos. Posteriormente, foram resfriados a temperatura ambiente e armazenados em sacos plásticos vedados até o momento das análises sensoriais. 


\subsection{Análise sensorial}

O presente estudo contou com 354 consumidores habituais de biscoito salgado (consomem biscoito ao menos uma vez na semana), destes 190 são do sexo feminino e 164 do sexo masculino, com idade entre 18 e 49 anos (21,19 $\pm 3,24$ anos).

A avaliação sensorial e a determinação dos limiares hedônicos (LAC e LRH) foram realizadas de acordo com os procedimentos de Lima Filho et al. (2015).

Os consumidores realizaram cinco sessões de testes de aceitação. Em cada sessão foram servidas duas amostras codificadas com números de três dígitos, uma era a amostra controle e a outra era uma das amostras estímulo, com teor reduzido de cloreto de sódio. As amostras foram servidas em ordem decrescente de concentração de cloreto de sódio, entre as sessões, e de forma aleatória, dentro de cada par. Os consumidores provaram as amostras da esquerda para a direita e informaram o quanto gostaram ou desgostaram de cada uma, por meio de ficha com escala hedônica de nove pontos (variando de $1=$ "desgostei extremamente" até $9=$ "gostei extremamente").

Para determinação do LAC, foi aplicado o teste t para amostras pareadas nas notas hedônicas da amostra controle e da amostra estímulo, de cada sessão. Foi elaborado um gráfico do valor t calculado (eixo Y1) em função da concentração de cloreto de sódio da amostra estímulo (eixo X) e ajustado um modelo de regressão. Para a seleção do modelo que melhor se ajustou aos dados foram avaliados a significância dos parâmetros da regressão e o coeficiente de determinação $\left(\mathrm{R}^{2}\right)$. No gráfico foi inserido uma linha tracejada referente ao valor t tabelado $(\alpha=5 \%)$, indicando o início da diferença significativa quanto a aceitação sensorial. O LAC foi calculado utilizando a equação do modelo, correspondendo a concentração de cloreto de sódio (variável x da equação) na qual o valor t calculado (variável y da equação) passa a ser igual ao valor t tabelado, ou seja, a concentração de cloreto de sódio em que começa a ocorrer redução significativa da aceitação sensorial do biscoito, devido à redução de cloreto de sódio.

Para determinação do LRH, no mesmo gráfico, inseriu-se um segundo eixo Y (Y2), referente às notas hedônicas médias das amostras estímulo, em função da concentração de cloreto de sódio (X). Também se ajustou um modelo de regressão aos dados experimentais, considerando os mesmos critérios para seleção do modelo do LAC. O ponto de corte foi representado no gráfico por uma linha tracejada, referente à nota hedônica 5 (termo hedônico “indiferente"), indicando o ponto de transição entre aceitação e rejeição sensorial do produto. O LRH foi calculado por meio da equação do modelo ajustado, sendo a concentração de 
Research, Society and Development, v. 9, n. 12, e36491211376, 2020

(CC BY 4.0) | ISSN 2525-3409 | DOI: http://dx.doi.org/10.33448/rsd-v9i12.11376

cloreto de sódio (variável x da equação) referente ao ponto no qual a nota hedônica média (variável y da equação) passa a ser igual a 5, ou seja, a concentração de cloreto de sódio em que começa a ocorrer rejeição sensorial do biscoito.

Para as análises estatísticas e a determinação dos limiares foi utilizado o software Microsoft Excel ${ }^{\circledR}$, versão 2016.

\section{Resultados e Discussão}

Na Figura 1 são apresentados os valores de t calculado (eixo Y1) e as notas hedônicas médias (eixo Y2) em função da concentração de cloreto de sódio das amostras estímulo (eixo $\mathrm{X})$. O modelo de regressão que melhor se ajustou aos valores t calculados foi o quadrático e o que melhor se ajustou as notas hedônicas médias foi o linear, ambos com parâmetros de regressão significativos $(\rho<0,05)$ e coeficiente de determinação superiores a 0,90 (Tabela 1$)$.

Figura 1 - Limiar de aceitação comprometida (LAC) para concentração de sódio em biscoito salgado.

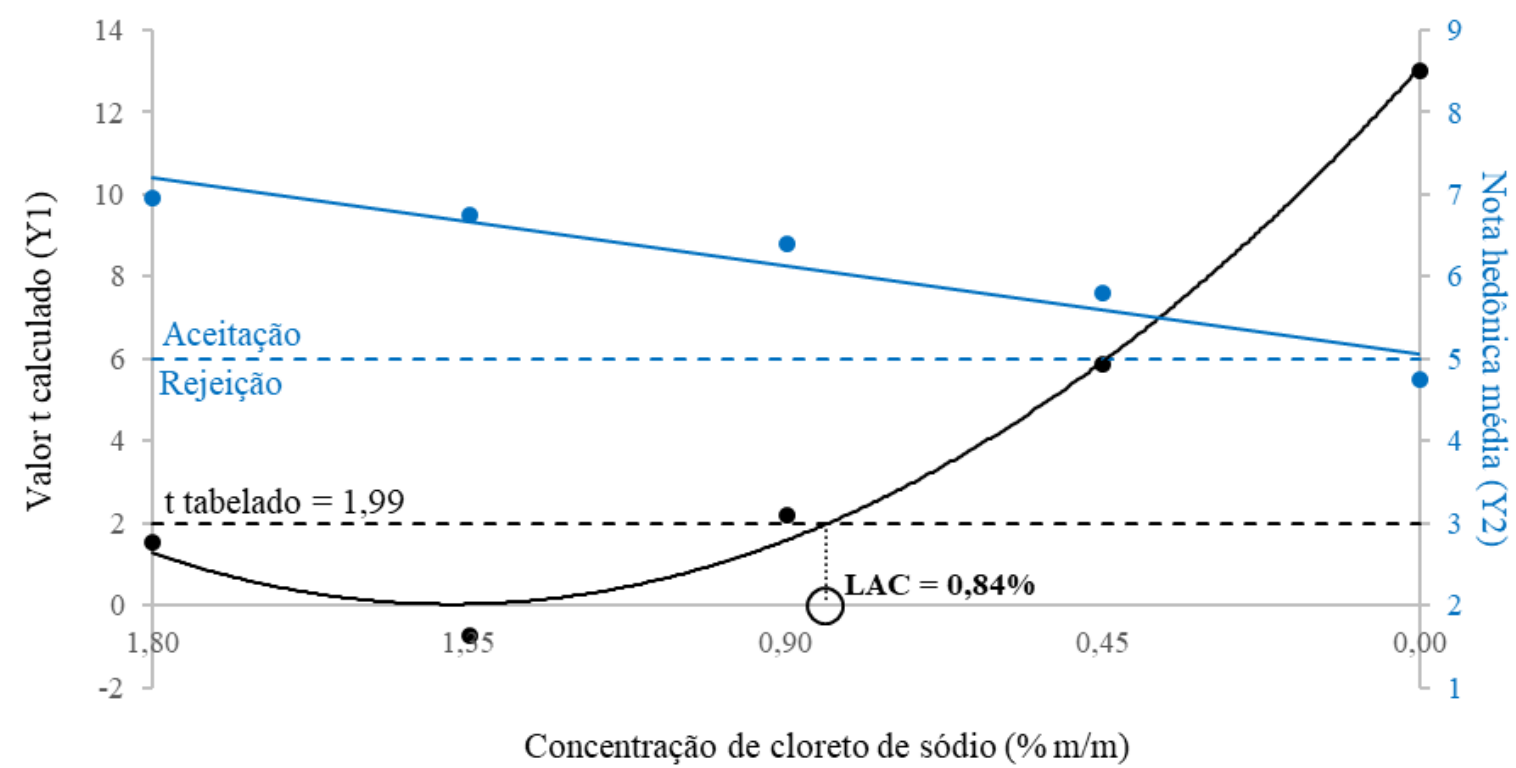

A linha tracejada preta representa o valor $\mathrm{t}$ tabelado a 5\% de significância para 353 graus de liberdade $(\mathrm{t}=1,99)$. A linha tracejada azul é referente à nota hedônica 5 , representando a transição entre aceitação e rejeição sensorial. Fonte: Autores. 
Research, Society and Development, v. 9, n. 12, e36491211376, 2020

(CC BY 4.0) | ISSN 2525-3409 | DOI: http://dx.doi.org/10.33448/rsd-v9i12.11376

Tabela 1 - Modelos ajustados e seus respectivos coeficientes de determinação $\left(R^{2}\right)$.

\begin{tabular}{ccc}
\hline & Modelo & $\mathrm{R}^{2}$ \\
\hline Equação 1 (LAC) & $\mathrm{Y} 1=6,912 \mathrm{x}^{2}-19,01 \mathrm{x}+13,092$ & 0,991 \\
Equação 2 (LRH) & $\mathrm{Y} 2=1,1902 \mathrm{x}+5,0599$ & 0,914
\end{tabular}

$\mathrm{Y} 1$ = valor $\mathrm{t}$ calculado; $\mathrm{Y} 2$ = nota hedônica média; $\mathrm{X}=$ concentração de cloreto de sódio $(\% \mathrm{~m} / \mathrm{m})$. Fonte: Autores.

O LAC foi calculado atribuindo-se o valor de 1,99 (t tabelado) para a variável Y1 da Equação 1 (Tabela 1), obtendo-se o valor de 0,84\% de cloreto de sódio, ou seja, a aceitação sensorial do biscoito salgado começa a reduzir significativamente somente a partir da redução da concentração de cloreto de sódio de 1,81\% para 0,84\% (g de cloreto de sódio em $100 \mathrm{~g}$ de biscoito), o que equivale a uma redução de 53,59\% no teor de cloreto de sódio. Logo, qualquer redução inferior a essa não compromete a aceitação sensorial do produto.

Para a determinação do LRH, deve-se substituir a variável Y2, da Equação 2, por 5 (nota referente ao termo hedônico "indiferente"), que representa o início da rejeição sensorial do produto. Por meio da equação ajustada aos dados experimentais, verificou-se que não ocorre rejeição sensorial devido à redução de cloreto de sódio do biscoito. No entanto, verificou-se que a amostra com $0 \%$ de $\mathrm{NaCl}$ apresentou média hedônica de 4,75, ou seja, foi rejeitada sensorialmente pelos consumidores. Portanto, apesar do modelo ajustado não permitir o cálculo do LRH, biscoitos sem adição de cloreto de sódio com concentração desse ingrediente próximo de $0 \%$ podem ser rejeitados sensorialmente pelos consumidores.

Analisando a Figura 1 e a Equação 2 também é possível verificar o efeito da variação da concentração de cloreto de sódio na aceitação sensorial do biscoito, sendo verificado uma menor aceitação sensorial à medida que o teor de cloreto de sódio reduzia.

Esses resultados são de grande interesse e aplicação pela indústria, uma vez que o LAC serve de parâmetro e pode ser utilizado para aumentar a ofertar de alimentos com reduzido teor de sódio, ou seja, mais saudáveis, sem afetar a aceitação pelo consumidor. Com a maior oferta de alimentos com reduzido teor de sódio e boa aceitação sensorial, menor tende a ser o consumo de sódio pelos consumidores e, consequentemente, menor a incidência de doenças crônicas não transmissíveis.

Muitos fatores de risco considerados preditivos para o desenvolvimento de doenças, em especial, de doenças crônicas não transmissíveis (DCNT), estão associados à hábitos alimentares e estilo de vida, entre eles pode-se citar o aumento da pressão arterial. A 
hipertensão é a causa mais frequente das demais doenças e condições crônicas, seu aparecimento é crescente e cada vez mais precoce, sendo o consumo excessivo de sódio o principal responsável pelo seu desenvolvimento (Brasil, 2011a). Projeções feitas por BibbinsDomingo et al. (2010) mostraram que reduzir em 3 gramas a ingestão diária de sal, leva a uma diminuição de 50\% nos casos de doenças cardiovasculares e reduz o número de mortes de 44 mil a 92 mil anualmente, sendo essa uma maneira eficiente e econômica para o controle dessa doença, quando comparado ao tratamento medicamentoso. Portanto, medidas e políticas públicas para redução da ingestão de sódio vem sendo aplicadas.

Diante de um cenário onde $75 \%$ do sal consumido pelos brasileiros provém de alimentos industrializados (Boscari \& Pereira, 2015), a diminuição do teor de sódio desses produtos é de muita relevância. Tendo isso em vista, os limiares determinados nesse estudo são de grande utilidade, pois torna possível a reformulação e produção de biscoitos salgados mais saudáveis e que se adequam às estratégias de redução de sódio mantendo a qualidade sensorial e atingindo as expectativas dos consumidores. Assim, o receio das indústrias em perder vendas devido à redução do teor de sódio de seus produtos passa a ser injustificável.

Uma das técnicas adotadas frequentemente na busca pela diminuição do teor de sódio em alimentos é a da redução de sódio sem percepção do consumidor, conhecida como "reduction by stealth". Essa técnica consiste na redução gradativa da quantidade de cloreto de sódio no produto, de forma que o consumidor se acostume com o alimento com menor teor de sal e não perceba sua redução na formulação (Paes \& Ravazi, 2018). Girgis et al. (2003), utilizando esse método, aplicou em pão branco reduções cumulativas de 5\% no teor de sódio a cada semana, durante 6 semanas consecutivas, e constatou que é possível uma diminuição de $25 \%$ da concentração de sódio nesse produto sem percepção pelo consumidor. Bernklau et al. (2017) verificaram que a redução de $\mathrm{NaCl}$ em $15 \%$ foi viável nos aspectos estruturais, de textura e sensoriais em massa de pizza fermentada. Reißner et al. (2019) estudaram a substituição de $\mathrm{NaCl}$ por diferentes sais isentos de sódio $\left(\mathrm{KCl}, \mathrm{MgCl}_{2}\right.$ e $\left.\mathrm{CaCl}_{2}\right)$ em pães, verificando que substituições de $50 \%$ de $\mathrm{NaCl}$ por $\mathrm{KCl}$ não alteram as características sensoriais dos pães, em relação à amostra controle (1,50 g de $\mathrm{NaCl}$ em $100 \mathrm{~g}$ de farinha).

No entanto, mesmo nas concentrações de cloreto de sódio em que ocorrem alteração do gosto salgado perceptível pelo consumidor, essa alteração inicial pode não ser o bastante para afetar a aceitação pelo consumidor. Como a aceitação sensorial pelo consumidor pode afetar as vendas do produto, as indústrias possuem grande interesse em descobrir as concentrações nas quais a redução de sódio resulta no início da alteração da aceitação sensorial do produto pelos consumidores, o que foi determinado no presente estudo. Para 
biscoito salgado, somente a partir de uma redução de 53,59\% de cloreto de sódio ocorre redução da aceitação sensorial de biscoito salgado.

Apesar desse grande interesse das indústrias nos valores dos limiares hedônicos, como a MLH é uma metodologia relativamente recente, ainda não existem muitos estudos determinando a partir de qual concentração a redução de cloreto de sódio resulta em redução da aceitação (LAC) e rejeição sensorial (LRH) de alimentos.

É importante ressaltar que a metodologia de determinação dos limiares hedônicos (LAC e LRH), utilizada no presente estudo, também pode ser aplicada na redução de sódio de outros alimentos e, dessa forma, será possível uma maior oferta de alimentos com teor de sódio reduzido e boa aceitação sensorial, auxiliando na diminuição do consumo de sódio pela população e suas consequências. Lima Filho et al. (2019) determinaram os limiares hedônicos para redução de sódio em hambúrguer bovino. Foi verificado que, partindo de uma amostra controle com $0,827 \%$ de sódio, começa a ocorrer comprometimento da aceitação sensorial do hambúrguer na concentração de $0,601 \%$ de sódio, ou seja, uma redução de $27,32 \%$ no teor de sódio; e começa a ocorrer rejeição sensorial do produto na concentração de $0.014 \%$ sódio, ou seja, redução de $98,31 \%$ no teor de sódio.

O presente estudo foi aplicado em consumidores de uma mesma região (Espírito Santo), os padrões de consumo podem variar entre as regiões e países, portanto, existe a necessidade de se realizar novos estudos a fim de verificar se as mesmas reduções no teor de cloreto de sódio podem ser obtidas com consumidores de outras regiões. Além disso, a maioria dos consumidores do presente estudo possuía faixa etária entre 18 e 30 anos. Os limiares sensoriais variam entre as faixas etárias, portanto, novos estudos podem ser realizados visando investir os valores dos limiares hedônicos para consumidores de outras faixas etárias. Novos estudos também podem ser realizados para determinar os limiares hedônicos para redução de cloreto de sódio em outros alimentos, informações que são de grande utilidade das indústrias de alimentos.

\section{Conclusão}

Concluiu-se que somente a partir de uma redução de 53,59\% no teor de cloreto de sódio $(1,81 \%$ para $0,84 \%)$ ocorre redução significativa na aceitação sensorial de biscoito salgado. Concluiu-se também que somente o biscoito sem adição de cloreto de sódio foi rejeitado sensorialmente pelo consumidor. Esses resultados são de grande interesse pela 
Research, Society and Development, v. 9, n. 12, e36491211376, 2020

(CC BY 4.0) | ISSN 2525-3409 | DOI: http://dx.doi.org/10.33448/rsd-v9i12.11376

indústria, uma vez que permite reduzir o teor de sódio de seus produtos sem afetar a aceitação sensorial e resultar em rejeição pelo consumidor.

\section{Agradecimentos}

À fundação de amparo à pesquisa e inovação do espirito santo (FAPES) e à coordenação de aperfeiçoamento de pessoal de nível superior (CAPES), pelo suporte financeiro.

\section{Referências}

ABIMAPI (Associação Brasileira das Indústrias de Biscoitos, Massas Alimentícias e Pães \& Bolos Industrializados). (2017). Anuário ABIMPAI 2017/2018 - Biscoitos. Recuperado de https://www.abimapi.com.br/anuario/pdf/Cap2.pdf

Alburto, N. J., Ziolkovska, A., Hooper, L., Elliot, P., Cappuccio, F. P. \& Meerpohl, J. J. (2013). Effect of lower sodium intake on health: Systematic review and metaanalyses. BMJ, 346, f1326. doi:10.1136/bmj.f1326

Bernklau, I., Neußer, C., Moroni, A. V., Gysler, C., Spagnolello, A., Chung, W., Becker, T. (2017). Structural, textural and sensory impact of sodium reduction on long fermented pizza. Food chemistry, 234, 398-407. doi:10.1016/j.foodchem.2017.04.188.

Bibbins-Domingo, K., Chertow, G. M., Coxson, P. G., Moran, A., Lightwood, J. M., Pletcher, M. J., \& Goldman, L. (2010). Projected Effect of Dietary Salt Reductions on Future Cardiovascular Disease. New England Journal of Medicine, 362(7), 590-599. doi:10.1056/NEJMoa0907355

Boscari, J. P. \& Pereira, F. B. (2015). Sódio em alimentos industrializados. In Anais do III Congresso de Pesquisa e Extensão da Faculdade da Serra Gaúcha (FSG). Caxias do Sul, RS, Brasil. Recuperado de http://ojs.fsg.br/index.php/pesquisaextensao/article/view/1596 
Brasil (2011b). Ministério da Saúde. Extrato de compromisso $\mathrm{n}^{\mathrm{o}} 34$ e $\mathrm{n}^{\mathrm{o}}$ 35, de 13 de novembro de 2011. Diário Oficial da República Federativa do Brasi, $n^{\circ}$ 247. Brasília - DF. Recuperado de http://189.28.128.100/dab/docs/portaldab/documentos/termo_5_dez_2011.pdf

Brasil (2011a). Ministério da Saúde, Secretaria de Vigilância em Saúde, Departamento de Análise de Situação de Saúde. Plano de ações estratégicas para o enfrentamento das doenças crônicas não transmissíveis (DCNT) no Brasil 2011-2022. Brasília - DF. Recuperado de https://bvsms.saude.gov.br/bvs/publicacoes/plano_acoes_enfrent_dent_2011.pdf

Brasil. (2015). Agência Nacional de Vigilância Sanitária. Teor de sódio nos alimentos processados. Informe técnico n. 65/2015. Recuperado de http://portal.anvisa.gov.br/documents/33916/388729/Informe+T\%C3\%A9cnico+n\%C2\%BA+ 69+de+2015/85d1d8f0-5761-4195-9aee-e992abd29b3e

Davidson, I. (2018). Biscuit, cookie and cracker production: process, production and packaging equipment. Academic Press. doi: 10.1016/C2017-0-03385-2

Girgis, S., Neal, B., Prescott, J., Prendergast, J., Dumbrell, S., Turner, C., \& Woodward, M. (2003). A one-quarter reduction in the salt content of bread can be made without detection. European Journal of Clinical Nutrition, 57(4), 616-620. doi:10.1038/sj.ejcn.1601583

Lima Filho, T., \& Minim, V. P. R. (2018). Limiares Afetivos. In V. P. R., MINIM (ed.). Análise sensorial: estudos com consumidores. (4a ed.), 11, p 325-343. Viçosa: Editora UFV.

Lima Filho, T., Della Lucia, S. M., Minim, L. A., Gamba, M. M., Lima, R. M. \& Minim, V. P. R. (2019). Directional hedonic thresholds for sodium concentration in hamburger. Food Quality and Preference, 78, 103722. doi:10.1016/j.foodqual.2019.103722

Lima Filho, T., Della Lucia, S. M., Minim, L. A., Silva, R. C. S. N., \& Minim, V. P. R. (2018) Validation of the hedonic threshold methodology in determining the hedonic rejection threshold. Journal of Sensory Studies, 33, 1-13. doi:10.1111/joss.12313

Lima Filho, T., Della Lucia, S. M., Minim, L. A; Silva, R. C. S. N., Silva, A. N., \& Minim, V. P. R. (2017). Validation of the hedonic threshold methodology in determining the 
compromised acceptance threshold. Journal of Sensory Studies, 32, 1-12. doi:10.1111/joss.12255.

Lima Filho, T., Minim, V. P. R., Silva, R. C. S. N., Della Lucia, S. M. \& Minim, L. A. (2015). Methodology for determination of two new sensory thresholds: Compromised acceptance threshold and rejection threshold. Food Research International, 76, 561-566. doi:10.1016/j.foodres.2015.07.037

Manley, D. (2000). Technology of biscuits, crackers and cookies (4a ed.), 493. Cambridge, England: Woodhead Publishing Limited.

Mozaffarian, D., Fahimi, S., Singh, G. M., Micha, R., Khatibzadeh, S., Engell, R. E., Lim, S., Danaei, G., Ezzati, M. \& Powles, J. Global burden of diseases nutrition and chronic diseases expert group. (2014). Global sodium consumption and death from cardiovascular causes. The New England Journal of Medicine, 371(7), 624-634. doi: 10.1056/NEJMoa1304127

Nielsen. (2014). Snack attack - What consumers are reaching for around the world. Recuperado de http://www.nielsen.com/content/dam/nielsenglobal/kr/docs/globalreport/2014/Nielsen\%20Global\%20Snacking\%20Report\%20September\%202014.pdf

Paes, J. S. \& Ravazi, R. (2018). Técnicas para redução de sódio nos alimentos industrializados. REGRAD - Revista Eletrônica De Graduação Do UNIVEM, 11(01), 379 390. Recuperado de https://revista.univem.edu.br/REGRAD/article/view/2626

Reißner, A. M., Wendt, J., Zahn, S., \& Rohm, H. (2019). Sodium-chloride reduction by substitution with potassium, calcium and magnesium salts in wheat bread. LWT, 108, 153159. doi:10.1016/j.jcs.2018.06.008.

World Health Organization - WHO (2014). WHO warns against the dangers of excessive salt consumption. Recuperado de https://www.who.int/westernpacific/news/detail/25-09-2014who-warns-against-the-dangers-of-excessive-salt-consumption 
Research, Society and Development, v. 9, n. 12, e36491211376, 2020

(CC BY 4.0) | ISSN 2525-3409 | DOI: http://dx.doi.org/10.33448/rsd-v9i12.11376

World Health Organization (2018). Noncommunicable diseases. Recuperado de https://www.who.int/westernpacific/news/detail/25-09-2014-who-warns-against-the-dangersof-excessive-salt-consumption

World Health Organization (2020). Salt Reduction. Recuperado de https://www.who.int/en/news-room/fact-sheets/detail/salt-reduction

Porcentagem de contribuição de cada autor no manuscrito

Laudiane Justo Sant'Anna - 19\%

Cirila Ionara Almeida Araújo - 16\%

Lara Lima Cornejo - $14 \%$

Eduardo da Silva Moreira - 10\%

Matheus Custódio de Paula - 7\%

Raquel Viera de Carvalho - 8\%

Suzana Maria Della Lucia - 10\%

Tarcísio Lima Filho - 16\% 